\title{
Qualidade de vida e voz: a autopercepção vocal de pessoas transgênero
}

\section{Quality of life and voice: the vocal self-perception of transgender people}

\author{
Rodrigo Dornelas ${ }^{1}$ (1), Raphaela Barroso Guedes-Granzotti ${ }^{2}$ (1), Alberto Silva Souza ${ }^{3}$ (1), \\ Ane Keslly Batista de Jesus ${ }^{3}$ (D), Kelly da Silva ${ }^{3}$ (D)
}

\begin{abstract}
RESUMO
Objetivo: Analisar o impacto da voz na qualidade de vida de pessoas transgênero (ou trans) e relacionar com a autopercepção vocal e a identidade de gênero. Métodos: Foram incluídos 27 indivíduos com idades entre 18 e 49 anos, usuários e usuárias do Ambulatório Trans de Sergipe: - "Portas abertas - Saúde integral das pessoas trans: cuidar e acolher", da Universidade Federal de Sergipe. Após anamnese, foram aplicados os instrumentos de Qualidade de Vida em Voz (QVV), o Transgender Voice Questionnaire (TVQ $\left.{ }^{\mathrm{MtF}}\right)$ e o Índice de Triagem para Distúrbio de Voz (ITDV). Foi observado maior número de homens trans $(63,0 \%)$, em relação ao de mulheres trans $(37,0 \%)$. A média de idade foi de 26,7 anos $( \pm 9,1)$ e $96,3 \%$ dos sujeitos possuíam uma queixa específica de voz. Resultados: O QVV apresentou um valor médio de 26,1 pontos para o escore total, o ITDV de 3,9 e o TVQ ${ }^{\mathrm{MtF}}$ de 70,6 , não havendo relação significativa entre os resultados e a identidade de gênero e a idade. Foi observada relação significativa entre o QVV e o $\mathrm{TVQ}^{\mathrm{MtF}}$. Conclusão: Observou-se uma baixa qualidade de vida em voz nas pessoas trans. Desta forma, quanto maior a percepção de suas alterações vocais, pior a sua qualidade de vida, independente do gênero com o qual se identificam (feminino ou masculino) e de possíveis distúrbios vocais.
\end{abstract}

Palavras-chave: Pessoas transgênero; Qualidade de vida; Qualidade da voz; Fonoaudiologia; Autoimagem

\begin{abstract}
Purpose: To analyze the impact of voice on the quality of life of transgender (or trans) people and to relate to vocal self-perception, gender identity. Methods: 27 individuals aged between 18 and 49 years, users of the Ambulatório Trans de Sergipe: - "Portas abertas - Saúde integral das pessoas trans: cuidar e acolher" - Universidade Federal de Sergipe" were included. After anamnesis, the Quality of Life in Voice (QLV) instruments, the Transgender Voice Questionnaire (TVQ $\left.{ }^{\mathrm{MtF}}\right)$ and the Voice Disorder Screening Index (VDSI) were applied. The highest number of trans men $(63.0 \%)$ was observed in relation to the group of trans women $(37.0 \%)$. The average age was 26.7 years $( \pm 9.1) .96 .3 \%$ had a specific voice complaint. Results: QLV presented a mean value of 26.1 points for total scores, the VDSI of 3.9 and TVQ ${ }^{\mathrm{MtF}}$ of 70.6, with no significant relationship between results and gender identity and age. A significant relationship was observed between QLV and TVQ ${ }^{\mathrm{MtF}}$. Conclusion: This study observed a poor quality of life in voice in trans people. Thus, the higher the perception of their vocal disorders, the worse their quality of life, regardless of gender (female or male) and possible vocal disorders.
\end{abstract}

Keywords: Transgender persons; Quality of life; Voice quality; Speech, Language and Hearing Sciences; Self concept

Trabalho realizado no Curso de Fonoaudiologia, Universidade Federal de Sergipe - UFS - Lagarto (SE), Brasil.

${ }^{1}$ Departamento de Fonoaudiologia, Universidade Federal do Rio de Janeiro - UFRJ - Rio de Janeiro (RJ), Brasil.

${ }^{2}$ Departamento de Fonoaudiologia, Universidade Federal de Sergipe - UFS - São Cristóvão (SE), Brasil.

${ }^{3}$ Departamento de Fonoaudiologia, Universidade Federal de Sergipe - UFS - Lagarto (SE), Brasil.

Conflito de interesses: Nada a declarar.

Contribuição dos autores: RD participou, na condição de orientador, da idealização do estudo, análise, interpretação dos dados e redação do artigo; RBGG participou da análise e interpretação dos dados e redação do artigo; ASS e AKBJ participaram da coleta, análise e interpretação dos dados e redação do artigo; KS participou da análise e interpretação dos dados e redação do artigo.

Financiamento: Nada a declarar.

Autor correspondente: Raphaela Barroso Guedes-Granzotti. E-mail: raphaelabgg@gmail.com

Recebido: Maio 29, 2019; Aceito: Fevereiro 13, 2020 


\section{INTRODUÇÃO}

A transexualidade, por envolver corpo, identidade e gênero e desafiar ideais da sociedade heteronormativa é um tema complexo $^{(1)}$, refletindo-se como uma experiência de conflito do ser com as normas de gênero, normas estas que definem que homens e mulheres de verdade possuem pênis e vagina e devem se comportar ativa e passivamente como tais e, assim, apenas a heterossexualidade daria sentido a essas diferenças anatômicas. Deste modo, cria-se uma associação em que o corpo remete ao sexo e gênero só existe quando associado a essa relação. Quando o gênero se manifesta fora dessa associação, é marginalizado e analisado como uma identidade transtornada e até passível de diagnóstico ${ }^{(2)}$.

Após anos de luta dos movimentos sociais, a Organização Mundial da Saúde (OMS), a atualização da Classificação Internacional das Doenças, CID-11, que substituiu a CID-10, retirou o termo transexualidade do capítulo sobre "transtornos de personalidade e comportamento", em um subcapítulo chamado "transtornos de identidade de gênero", para integrar um novo capítulo, intitulado "condições relacionadas à saúde sexual" e classificado como "incongruência de gênero". Assim, a transexualidade não está mais relacionada a uma escolha ou a uma doença mental, passando a ser considerada uma questão de identidade ${ }^{(3)}$.

A voz desempenha um papel fundamental no processo de construção dessa nova identidade, seja na autoaceitação ou na receptividade social ${ }^{(4)} \mathrm{e}$, a autoimagem, mensurada por meio de instrumentos que auxiliam a percepção do sujeito quanto à sua voz, faz parte deste processo ${ }^{(5)}$. É essencial, portanto, incluir a perspectiva do indivíduo, quando se avaliam os resultados dos procedimentos executados, seja a terapia vocal, cirurgia ou tratamento medicamentoso ${ }^{(6)}$.

Homens trans são pessoas que reivindicam o reconhecimento social e legal como homens, sendo que alguns também se denominam transhomens, ou female-to-male $(\mathrm{FtM})^{(3)}$. Geralmente, nas modificações vocais, são beneficiados com o tratamento hormonal, requerendo menos estratégias, como cirurgias ou acompanhamento fonoaudiológico.

As mulheres trans são pessoas que reivindicam o reconhecimento social e legal como mulheres, ou transmulheres, ou male-to-female $(\mathrm{MtF})^{(3)}$. Nestes casos, a obtenção de uma voz feminina é uma meta altamente desejável, porém difícil de ser alcançada, na maioria dos casos, dependendo muito do início do processo de transição, pois, quanto mais próximo do processo de muda vocal, melhores serão os resultados ${ }^{(7)}$. Estratégias como entonação, articulação e linguagem podem auxiliar nesse processo de construção vocal, tanto para homens trans, como para mulheres trans ${ }^{(8,9)}$.

Historicamente, em função das necessidades de saúde da população, a Fonoaudiologia buscou ampliar suas formas de atendimento, modificando as práticas que tiveram bases biomédicas, em que se valorizava apenas a doença e a reabilitação individual, para uma prática de aproximação entre a Fonoaudiologia e os ideais de Educação em Saúde, considerando a saúde como um direito de todos e dando funcionalidade aos princípios de universalidade e equidade do SUS ${ }^{(10)}$.

Dentro desta concepção, o fonoaudiólogo deve fazer parte da equipe multidisciplinar do processo transexualizador, que tem como foco a melhor qualidade de vida da população trans, sendo a voz um importante aspecto na percepção de gênero do sujeito $^{(11)}$. Deste modo, o presente estudo teve o objetivo de analisar o impacto da voz na qualidade de vida de pessoas trans e relacionar com a autopercepção vocal e a identidade de gênero.

\section{MÉTODO}

O estudo de caráter observacional, transversal e analítico, de amostragem por conveniência, foi aprovado pelo Comitê de Ética em Pesquisa (CEP) da Universidade Federal de Sergipe $\left(\mathrm{n}^{\circ} 1.313 .343\right)$. Todas as pessoas que concordaram em participar, mediante explicação prévia, assinaram o Termo de Consentimento Livre e Esclarecido, conforme Resolução 466/2012-CNS/MS.

Foram convidados a participar todos os usuários que estavam aguardando o primeiro atendimento na sala de espera do Ambulatório Trans de Sergipe: "Portas abertas - Saúde integral das pessoas trans: cuidar e acolher", no período de janeiro a agosto de 2017. Foram excluídas do estudo pessoas com idade inferior a 18 anos, aquelas que haviam iniciado o processo transexualizador assistido por profissional de saúde, naquele serviço ou em outro, ou que haviam realizados procedimentos cirúrgicos relacionados, ou terapia fonoaudiológica com vistas à readequação vocal.

Assim, foram incluídas 27 pessoas, sendo 17 homens trans (gênero masculino) e 10 mulheres trans (gênero feminino), com média de idade de $26,7( \pm 9,1)$ anos.

$\mathrm{O}$ ambulatório onde foi realizada a pesquisa é multiprofissional e disponibiliza atendimento em Fonoaudiologia, Psicologia, Nutrição, Endocrinologia, Psiquiatria, Ginecologia e Terapia Ocupacional.

No primeiro momento, foi realizada uma breve anamnese com informações quanto ao nome, idade, gênero e presença, ou não, de queixa vocal. Em seguida, foram aplicados três instrumentos: Qualidade de Vida em Voz (QVV), Transgender Voice Questionnaire $\left(\mathrm{TVQ}^{\mathrm{MtF}}\right)$ e Índice de Triagem para Distúrbio de Voz (ITDV).

O QVV foi traduzido e validado para o português brasileiro por Gasparini e Behlau ${ }^{(11)}$ e tem como objetivo quantificar o impacto que determinada alteração vocal traz para a qualidade de vida do sujeito ${ }^{(12)}$. Possui dez itens, distribuídos em seis questões físicas e quatro socioemocionais. Cada item pode ser classificado numa escala de cinco pontos, em que 1 "não é um problema" e 5 "é um problema muito grande". Quanto maior a pontuação calculada, melhor é a qualidade de vida do sujeito. Para o cálculo dos escores, utiliza-se um algoritmo padrão, que pode variar de 0 a 100 , este último indicando a melhor qualidade de vida possível.

O TVQ ${ }^{\mathrm{MtF}}$ é um questionário de autopercepção, específico para pessoas trans, desenhado para medir a percepção desta população quanto a sua $\mathrm{voz}^{(13)}$. Este questionário, traduzido e adaptado para o português brasileiro, é composto de 30 itens e possui uma escala de classificação que varia de 1 a 4 , sendo 1 = "nunca ou raramente", 2 = "algumas vezes", 3 = "frequentemente" e 4 = "usualmente ou sempre". A pontuação varia de 30 a 120, mínimo e máximo, respectivamente ${ }^{(14)}$. Quanto maior a pontuação, pior é a percepção sobre a própria voz.

Utilizou-se, ainda, um questionário adaptado com base no $\mathrm{TVQ}^{\mathrm{MtF}}$. Por se tratar de um instrumento específico para mulheres trans, houve uma adaptação para que abrangesse ambos os gêneros, tanto mulheres como homens trans. A adaptação se deu, exclusivamente, com a mudança de palavras, termos e expressões remetidos ao gênero feminino por demais, em 
comparação ao gênero masculino, a fim de abarcar, também, este gênero.

O ITDV é um instrumento de autopercepção, elaborado e validado por Ghirardi et al. ${ }^{(15)}$, composto por 12 sintomas vocais comuns e possui uma escala de classificação dividida em "nunca", "raramente", "às vezes" e "sempre". Para as respostas "às vezes" ou "sempre", soma-se 1 ponto. A nota de corte para a presença de um distúrbio vocal é de 5 pontos.

Foram utilizados os softwares Excel e SPSS ${ }^{\circledR}$ 16.0, para análise dos dados. A normalidade dos dados foi estudada por meio do teste de Kolmogorov-Smirnov, sendo uma amostra paramétrica. Para verificação de correlação entre os resultados da pontuação total entre os instrumentos (ITDV, QVV e TVQ ${ }^{\mathrm{MtF}}$ ), para verificação da correlação da idade nos resultados dos instrumentos, foi utilizado o teste de correlação bivariada de Pearson (r). Foram estipulados como sendo de correlação fraca valores de $\mathrm{r}$ entre 0 e 0,3 ; moderada entre 0,4 e 0,6 e forte, quando maior que $0,7^{(16)}$. Para comparação das médias dos instrumentos aplicados (ITDV, QVV e TVQ ${ }^{\mathrm{MtF}}$ ) entre os gêneros foi utilizado o teste $\mathrm{t}$ Student para amostras independentes. Foi estabelecido como estatisticamente significativo o valor de $\mathrm{p}$ de $5 \%(\mathrm{p}<0,05)$.

\section{RESULTADOS}

Na entrevista inicial, $26(96,3 \%)$ pessoas declararam apresentar alguma queixa vocal especifica. O teste t Student para amostras independentes não evidenciou diferenças significativas entre mulheres e homens trans, nas respostas do ITDV $(p=0,6)$, $\operatorname{TQV}^{\mathrm{MtF}}(\mathrm{p}=0,4)$ e do QVV $(\mathrm{p}=0,7)$ (Tabela 1$)$.

Os resultados referentes ao ITDV demonstraram que $12(44,4 \%)$ pessoas apresentaram resultados sugestivos de distúrbios vocais (pontuação final igual ou maior que 5), sendo a média encontrada $34,1( \pm 3,1)$. O QVV, no domínio socioemocional, apresentou média de $60,6( \pm 25,7)$ pontos $(90,65$ foi a melhor pontuação para este domínio) e, no domínio físico, média de $64,8( \pm 28,9)$ pontos $(89,60$ foi a melhor pontuação para este domínio), indicando maior comprometimento dos aspectos socioemocionais. No TQV ${ }^{\mathrm{MtF}}$, a média obtida foi de 70,6 $( \pm 24,7)$ (valor mínimo de resposta igual a 30 pontos e máximo de 120 pontos).
Em relação aos resultados da correlação bivariada de Pearson entre os resultados dos testes ITDV, QVV (físico, emocional e total) e TQV ${ }^{\mathrm{MtF}}$, houve correlação significativa de grau forte entre o TQV ${ }^{\mathrm{MtF}}$ e QVV socioemocional, entre o TQV ${ }^{\mathrm{MtF}}$ e QVV físico e entre o TQV ${ }^{\mathrm{MtF}}$ e pontuação total, indicando que, quanto maior a qualidade de vida em voz menor é a percepção de sua própria voz.

A Tabela 2 ilustra as pontuações totais médias nos testes ITDV, QVV e TQV ${ }^{\mathrm{MtF}}$ e os resultados da correlação bivariada de Pearson entre os resultados dos testes ITDV, QVV (físico, emocional e total) e TQV ${ }^{\mathrm{MtF}}$.

\section{DISCUSSÃO}

O presente estudo é relevante para que se possa conhecer a autopercepção vocal de pessoas trans e, assim, identificar possíveis impactos sobre a qualidade de vida dessa população.

A população trans é composta, em sua maioria, por mulheres trans, cerca de duas para um, em relação aos homens trans. Entretanto, no presente estudo, a maioria da amostra foi composta por homens trans, ratificando os achados de um estudo conduzido no Irã, que também obteve a maioria pertencente a este gênero $^{(16)}$, o que pode indicar tendência no aumento da procura de assistência à saúde por parte da população masculina trans ${ }^{(14)}$.

Além disso, outro achado nesse mesmo estudo ${ }^{(14)}$, que pode indicar tendência à mudança no perfil da população trans que está procurando atendimento, foi a média de idade da população estudada, demonstrando que as pessoas trans têm buscado mais cedo seus direitos, dentre eles, o acesso à saúde. A média foi de dez anos a menos do que a idade encontrada em um estudo conduzido na Espanha ${ }^{(17)}$, de 37,3 anos. A procura tardia por atendimento à saúde, por parte dessa população, origina-se do estigma e do preconceito social ${ }^{(18)}$, tendência que pode estar mudando, em decorrência do número crescente de movimentos políticos ${ }^{(19)}$ e sociais que visam o empoderamento das pessoas $\operatorname{trans}^{(20)}$.

Nesse contexto, compreender as queixas e os comportamentos sociais relacionados à voz de pessoas trans é necessário para o planejamento das ações de saúde voltadas a essa população. No presente estudo, quase a totalidade dos participantes referiram alguma queixa vocal na anamnese. As queixas apresentadas por

Tabela 1. Autopercepção vocal em homens e mulheres trans

\begin{tabular}{lccccc}
\hline & ITDV & QVV Físico & QVV Socioemocional & QVV escore & TQV \\
\hline Homem Trans & $3,9 \pm 3,2$ & $65,4 \pm 29,2$ & $63,2 \pm 25,3$ & $64,1 \pm 26,1$ & $67,5 \pm 24,6$ \\
Mínimo-máximo & $0-11$ & $6,2-100$ & $12,5-100$ & $10-100$ & $30-103$ \\
Mulher Trans & $4,3 \pm 2,9$ & $63,7 \pm 30$ & $56,2 \pm 27,0$ & $59,2 \pm 27,1$ & $75,9 \pm 25,2$ \\
Mínimo-máximo & $0-8$ & $0-100$ & $16,7-100$ & $10-100$ & $32-114$ \\
Pvalor & $\mathrm{p}=0,6$ & $\mathrm{p}=0,9$ & $\mathrm{p}=0,5$ & $\mathrm{p}=0,7$ & $\mathrm{p}=0,4$ \\
\hline
\end{tabular}

Teste t-Student para amostras independentes

Legenda: ITDV = Índice de Triagem para Distúrbio de Voz; QVV = Qualidade de Vida em Voz; TQV = Transgender Voice Questionnaire

Tabela 2. Resultados de correlação entre os resultados do Índice de Triagem para Distúrbio de Voz, Qualidade de Vida em Voz e Transgender Voice Questionnaire

\begin{tabular}{ccccc}
\hline & \multirow{2}{*}{ ITDV } & \multicolumn{1}{c}{ QVV } & & \\
\cline { 3 - 5 } & & Físico & Socioemocional & Escore total \\
\hline ITDV & --- & $r=-0,1 ; p=0,3$ & $r=-0,2 ; p=0,2$ & $r=-0,2 ; p=0,4$ \\
TQV & $r=0,3 ; p=0,2$ & $r=-0,5 ; p=0,01^{*}$ & $r=-0,6 ; p=0,01^{*}$ & $r=-0,6 ; p=0,01^{*}$ \\
\hline
\end{tabular}

Teste de correlação de Pearson; *Valores estatisticamente significativos $(p<0,05)$

Legenda: ITDV = Índice de Triagem para Distúrbio de Voz; QVV = Qualidade de Vida em Voz; TQV = Transgender Voice Questionnaire 
pessoas trans podem estar relacionadas, ou não, a redesignação sexual e, quando presentes, podem influenciar diretamente no aspecto psicossocial da $\mathrm{voz}^{(13,21)}$.

Embora o ITDV tenha sido idealizado para a utilização em profissionais da voz, optou-se em utilizá-lo nesta pesquisa, por ser um instrumento que investiga a presença de sintomas vocais comuns em toda a população, quais sejam: rouquidão, falha na voz, pigarro, cansaço ao falar, entre outros ${ }^{(15)}$. Neste estudo, $12(44,4 \%)$ pessoas apresentaram risco para distúrbio vocal, sugeridos por escore maior que 5 pontos $^{(15)}$. O ITDV apresenta um alto grau de sensibilidade para mapeamento de distúrbios da voz. Portanto, sugere-se que, caso o sujeito faça referência a cinco ou mais sintomas vocais, seja encaminhado para avaliação otorrinolaringológica e fonoaudiológica ${ }^{(15)}$.

O surgimento dessas alterações vocais pode ser justificado quando se considera a voz como um importante fator na percepção de gênero ${ }^{(14)}$, fazendo com que pessoas trans procurem modelar sua voz ou realizar esforços espontâneos para adequá-la a uma categoria social ${ }^{(22,23)}$. Esse esforço na produção da voz, assim como adaptações negativas, pode ser responsável por disfonias ${ }^{(24,25)}$.

Com relação ao $\mathrm{TVQ}^{\mathrm{MtF}}$, apesar de ser um instrumento criado especificamente para mulheres trans ${ }^{(14)}$, pôde-se perceber que não houve diferença significativa para as médias de pontuações entre homens e mulheres trans, demonstrando ser um instrumento válido para uso em ambos os gêneros, com a necessidade apenas de algumas adaptações na linguagem.

A avaliação vocal é essencial em pessoas trans, principalmente por instrumentos de autopercepção, visto que a voz se relaciona com o gênero e que está diretamente ligada à qualidade de vida ${ }^{(13)}$. Os escores médios obtidos na aplicação do TVQ ${ }^{\mathrm{MtF}}$, neste estudo, foram visivelmente superiores (ou seja, a autopercepção vocal de pessoas trans indicou maiores prejuízos para o indivíduo) aos encontrados em outro estudo com pessoas trans brasileiras ${ }^{(18)}$, que concluiu que indivíduos vivenciavam o estresse por se identificarem com o sexo oposto, ou por estarem insatisfeitos com suas vozes. Com relação às mulheres trans, foi observado que, quanto maior o tempo de apresentação como mulheres, menores os escores obtidos. Os autores sugeriram que, quanto maior o tempo de vivência trans, maior é a construção singular sobre a mulher que deseja ser ${ }^{(13)}$.

A qualidade de vida refere-se a um conjunto de fatores físicos, mentais e sociais que interferem na vivência de um sujeito. Assim, compreende-se que uma alteração vocal pode implicar a qualidade de vida, dependendo das particularidades de cada sujeito(26). Os resultados apresentados no QVV, nesta pesquisa, demonstraram que as vozes da população trans estudada têm um alto impacto negativo na qualidade de vida, com valor médio de 26,1 pontos para o escore total, valor este muito inferior ao valor de corte descrito na literatura, que apontou que um escore total menor ou igual a 60 implicam um alto impacto da voz na qualidade de vida ${ }^{(27)}$.

O impacto negativo na qualidade de vida da população estudada ficou mais evidente quando observada a média individualizada do domínio socioemocional e do domínio físico, que foi, respectivamente $9,6( \pm 4,2)$ e $16,5( \pm 5,9)$. A nota de corte estipulada é de 91,2 pontos $^{(28)}$. Valores abaixo indicariam que as alterações vocais percebidas impactam a qualidade de vida, ou seja, o presente estudo identificou um forte impacto na vida das pessoas trans, devido a sua voz.

Os dados observados nesta pesquisa, quanto à qualidade de vida em voz da população trans, reforçam a ideia da importância fundamental que os aspectos vocais ocupam no processo transexualizador de construção de uma nova identidade, seja na própria autoaceitação, ou na aceitação do meio social, sendo que a voz deve ser considerada um elemento essencial na identificação do gênero.

Quando analisados os resultados da avaliação do QVV com o TVQ ${ }^{\mathrm{MtF}}$, encontrou-se correlação de grau forte, negativa e significativa, entre eles. Os resultados apontaram que, quanto maior a pontuação do $\mathrm{TVQ}^{\mathrm{MtF}}$, menor a pontuação no QVV, ou seja, quanto maior a percepção dos problemas mensuráveis pelo TVQ ${ }^{\mathrm{MtF}}$ pior a qualidade de vida em voz. Ou seja, quanto maior a percepção e a sensibilidade aos problemas vocais e sua interferência nas atividades diárias, maior o impacto na qualidade de vida das pessoas trans.

A autopercepção obtida por meio do ITDV, entretanto, não encontrou relação com o QVV. Este dado pode sugerir algumas questões específicas e divergentes entre o ITDV e o $\mathrm{TVQ}^{\mathrm{MtF}}$, visto que o ITDV, idealizado como instrumento de triagem para distúrbios vocais, investiga a sintomatologia vocal, principalmente considerando as lesões laríngeas, enquanto o $\mathrm{TVQ}^{\mathrm{MtF}}$ integra questões da avaliação multidimensional da voz e da comunicação, considerando questões identitárias da população trans.

Considera-se, então, que o $\mathrm{TVQ}^{\mathrm{MtF}}$ é um questionário de autopercepção vocal, específico para pessoas trans e, assim, os achados deste estudo trazem reflexões relevantes, como a necessidade de utilização de instrumentos de autopercepção vocal específicos para esta população, que considerem as suas singularidades relacionadas às características e demandas em relação à identidade e conduta de gênero.

Apesar de algumas conquistas na última década, a população trans ainda convive com uma realidade caracterizada pela extrema marginalização e exclusão social, o que evidencia a necessidade de que as políticas públicas invistam, de forma consistente e contínua, no enfrentamento do estigma e das condições de exclusão social que marcam o cotidiano desta população. Reforça-se, ainda, a importância de se considerar, no processo de construção de programas e ações, tanto a capacidade de atuação desses sujeitos, como os contextos de vulnerabilidade e os problemas de ordem estrutural da rede pública de saúde ${ }^{(29)}$.

Tendo em vista que a voz é um importante aspecto na percepção de gênero do sujeito e está diretamente relacionada à qualidade de vida das pessoas, salienta-se que estudos como este, que avaliam a autopercepção vocal de pessoas trans, ainda escassos na literatura brasileira, podem servir como um parâmetro importante na avaliação das politicas públicas e na elaboração de protocolos específicos voltadas para essa população.

\section{CONCLUSÃO}

Observou-se uma baixa qualidade de vida em voz nas pessoas trans. Desta forma, quanto maior a percepção de suas alterações vocais, pior a sua qualidade de vida, independente do gênero que se identificam (feminino ou masculino) e de possíveis distúrbios vocais.

\section{REFERÊNCIAS}

1. Silva AL, Oliveira AAS. Transexualidade/travestilidade na literatura brasileira: sentidos e significados. Arq Bras Psicol. 2013;65(2):274-87. 
2. Bento B. A reinvenção do corpo: sexualidade e gênero na experiência transexual. Rio de Janeiro: Garamond; 2006.

3. Jesus JG. Orientações sobre a população transgênero: conceitos e termos. Brasília: Autor, 2012

4. Pérez Alvarez JC. Voice and identity in transsexuality. Handchir Mikrochir Plast Chir. 2011;43(4):246-9. PMid:21833878.

5. Costa EBM, Pernambuco LA. Autoavaliação vocal e avaliação perceptivoauditiva da voz em mulheres com doença tireoidiana. Rev CEFAC. 2014;16(3):967-73. http://dx.doi.org/10.1590/1982-021620145913.

6. Dacakis G, Oates J, Douglas J. Beyond voice: perceptions of gender in male-to-female transsexuals. Curr Opin Otolaryngol Head Neck Surg. 2012;20(3):165-70. http://dx.doi.org/10.1097/MOO.0b013e3283530f85. PMid:22487788.

7. Gorham-Rowan M, Morris R. Aerodynamic analysis of male-tofemale transgender voice. J Voice. 2006;20(2):251-62. http://dx.doi. org/10.1016/j.jvoice.2005.03.004. PMid:16112545.

8. Van Borsel J, Cayzeele M, Heirman E, T'sjoen G. Conversational topics in transsexual persons. Clin Linguist Phon. 2014;28(6):428-36. http://dx.doi.org/10.3109/02699206.2013.875594. PMid:24446798.

9. Hancock A, Colton G, Douglas F. Intonation and gender perception: applications for transgender speakers. J Voice. 2014;28(2):203-9. http://dx.doi.org/10.1016/j.jvoice.2013.08.009. PMid:24094799.

10. Dornelas R, Sousa MF, Mendonça AVM. Mendonça. Informação, educação e comunicação em saúde: análise das concepções dos coordenadores das campanhas de voz no Distrito Federal. Rev CEFAC. 2014 JanFev;16(1):274-82. http://dx.doi.org/10.1590/1982-0216201412212.

11. Gasparini G, Behlau M. Quality of life: validation of the Brazilian Version of the Voice-Related Quality of Life (V-RQOL) measure. J Voice. 2009;23(1):76-81. http://dx.doi.org/10.1016/j.jvoice.2007.04.005. PMid:17628396.

12. Hogikyan ND, Sethuraman G. Validation of an Instrument to Measure Voice-Related Quality of Life (V-RQOL). J Voice. 1999;13(4):557-69. http://dx.doi.org/10.1016/S0892-1997(99)80010-1. PMid:10622521.

13. Dacakis G, Davies S, Oates JM, Douglas JM, Johnston JR. Development and Preliminary Evaluation of the Transsexual Voice Questionnaire for Male-to-Female Transsexuals. J Voice. 2013;27(3):312-20. http:// dx.doi.org/10.1016/j.jvoice.2012.11.005. PMid:23415146.

14. Santos HHANM, Aguiar AGO, Baeck HE, Van Borsel J. Translation and preliminary evaluation of the Brazilian Portuguese version of the Transgender Voice Questionnaire for male-to-female transsexuals. CoDAS. 2015;27(1):89-96. http://dx.doi.org/10.1590/2317-1782/20152014093. PMid:25885202.

15. Ghirardi ACAM, Ferreira LP, Giannini SPP, Latorre MRDO. Screening Index for Voice Disorder (SIVD): development and Validation. J Voice. 2013;27(2):195-200. http://dx.doi.org/10.1016/j.jvoice.2012.11.004. PMid:23280383.

16. Saeidzadeh Z. Transsexuality in Contemporary Iran: Legal and Social Misrecognition. Fem Leg Stud. 2016;24(3):249-72. http://dx.doi. org/10.1007/s10691-016-9332-x.
17. Basterra V, Ruiz R, Toni M, Rebolé A, Pérez de Mendiola Y, Forga L. Estudio descriptivo de la transexualidad en Navarra. An Sist Sanit Navar. 2012;35(3):455-60. http://dx.doi.org/10.4321/S113766272012000300011. PMid:23296226.

18. Schwarz K, Fontanari AMV, Mueller A, Costa AB, Soll B, da Silva DC, et al. Transsexual Voice Questionnaire for Male-to-female Brazilian Transsexual People. J Voice. 2017 Jan;31(1):120.e15-20. http://dx.doi. org/10.1016/j.jvoice.2016.02.012. PMid:27146028.

19. Brasil. Ministério da Saúde. Secretaria de Gestão Estratégica e Participativa. Departamento de Apoio à Gestão Participativa. (2013). Política nacional de saúde integral de lésbicas, gays, bissexuais, travestis e transexuais. 1. ed. Brasília. 32 p.

20. Almeida G, Murta D. Reflexões sobre a possibilidade da despatologização da transexualidade e a necessidade da assistência integral à saúde de transexuais no Brasil. Sex, Salud Soc. 2013;(14):380-407.http://dx.doi. org/10.1590/S1984-64872013000200017.

21. Dornelas R, Granzotti RBG, Leite AFS, Silva K. A redesignação vocal em pessoas trans. CoDAS. 2017;29(2):e20160168. http://dx.doi. org/10.1590/2317-1782/20172016168. PMid:28300938.

22. Bergel S. Voz do transexual masculino [monografia]. Porto Alegre: Centro de Especialização em Fonoaudiologia Clínica; 1999.

23. Drumond LB. Fonoaudiologia e transgenitalização: a voz no processo de reelaboração da identidade social do transexual. In: Anais do XV Encontro Nacional da Associação Brasileira de Psicologia Social; 2009, Maceió. Maceió: ABRAPSO; 2009.

24. Davies J, Anderson S, Huchison L, Stewart G. Interactions between voice clinics and singing teachers: a report on the British Voice Association questionnaire to voice clinics in the UK. Logoped Phoniatr Vocol. 2007;32(2):83-6. http://dx.doi.org/10.1080/14015430601114849. PMid: 17613790

25. Silva LP, Gomes AC, Lucena JA, Araújo ANB. Estratégias de enfrentamento da disfonia na perspectiva biopsicossocial. In: Anais do XXIII Congresso Brasileiro e IX Congresso Internacionalde Fonoaudiologia; 2015; Salvador. São Paulo: SBFa; 2015. p. 7-9.

26. Spina AL, Maunsell R, Sandalo K, Gusmão R, Crespo A. Correlação da qualidade de vida e voz com atividade profissional. Rev Bras Otorrinolaringol. 2009;75(2):275-9. http://dx.doi.org/10.1016/S18088694(15)30790-4.

27. Servilha EAM, Roccon PF. Relação entre voz e qualidade de vida em professores universitários. Rev CEFAC. 2009 Jul-Set;11(3):440-8. http://dx.doi.org/10.1590/S1516-18462009005000029.

28. Behlau M, Madazio G, Moreti F, Oliveira G, Dos Santos LM, Paulinelli $\mathrm{BR}$, et al. Efficiency and cutoff values of self-assessment instruments on the impact of a voice proble. J Voice. 2016 Jul;30(4):506.e9-18. http://dx.doi.org/10.1016/j.jvoice.2015.05.022. PMid:26168902.

29. Monteiro S, Brigeiro M, Barbosa RM. Saúde e direitos da população trans. Cad Saude Publica. 2019;35(4):e00047119. http://dx.doi. org/10.1590/0102-311x00047119. PMid:30994736. 